\title{
Evidence of cAMP involvement in cellobiohydrolase expression and secretion by Trichoderma reesei in presence of the inducer sophorose
}

\author{
Karoline Maria Vieira Nogueira', Mariana do Nascimento Costa ${ }^{1}$, Renato Graciano de Paula ${ }^{1}$, \\ Flávia Costa Mendonça-Natividade ${ }^{2}$, Rafael Ricci-Azevedo ${ }^{2}$ and Roberto Nascimento Silva ${ }^{1 *}$
}

\begin{abstract}
Background: The signaling second messenger cyclic AMP (CAMP) regulates many aspects of cellular function in all organisms. Previous studies have suggested a role for CAMP in the regulation of gene expression of cellulolytic enzymes in Trichoderma reesei (anamorph of Hypocrea jecorina).

Methods: The effects of cAMP in T. reesei were analyzed through both activity and expression of cellulase, intracellular cAMP level measurement, western blotting, indirect immunofluorescence and confocal microscopy.

Results: To elucidate the involvement of CAMP in the cellulase expression, we analyzed the growth of the mutant strain $\triangle a c y 1$ and its parental strain QM9414 in the presence of the inducers cellulose, cellobiose, lactose, or sophorose, and the repressor glucose. Our results indicated that cAMP regulates the expression of cellulase in a carbon source-dependent manner. The expression cel7a, and cel6a genes was higher in the presence of sophorose than in the presence of cellulose, lactose, cellobiose, or glucose. Moreover, intracellular levels of cAMP were up to four times higher in the presence of sophorose compared to other carbon sources. Concomitantly, our immunofluorescence microscopy and western blot data suggest that in the presence of sophorose, cAMP may regulate secretion of cellulolytic enzymes in $T$. reesei.

Conclusions: These results allow us to better understand the role of CAMP and expand our knowledge on the signal transduction pathways involved in the regulation of cellulase expression in T. reesei. Finally, our data may help develop new strategies to improve the expression of cel7a and cel6a genes, and therefore, favor their application in several biotechnology fields.
\end{abstract}

Keywords: Carbon source, Cellulases, Saprophytic fungus, Secondary messenger, Signaling pathways

\section{Background}

Trichoderma reesei (anamorph of Hypocrea jecorina) is a saprophytic fungus that has an efficient secretion system of cellulolytic proteins involved in the degradation of cell wall polysaccharides of plants. This fungus has an enormous ability to both produce and secrete cellulases. This makes it the most important industrial fungus for the production of these enzymes, which are used for several purposes including the production of bioethanol in the biofuel industry [1].

\footnotetext{
* Correspondence: rsilva@fmrp.usp.br

'Department of Biochemistry and Immunology, Ribeirão Preto Medical School, University of São Paulo, 14049-900 Ribeirão Preto, SP, Brazil Full list of author information is available at the end of the article
}

The cellulolytic system of this fungus consists of at least three different types of enzymes-i.e., exoglucanases (cellobiohydrolases EC 3.2.1.91), endoglucanases (EC 3.2.1.4), and $\beta$-glucosidases (EC 3.2.1.21) [2] — that act synergistically and are coordinately expressed in specific growing conditions $[3,4,5]$.

In general, fungi belonging to the Trichoderma genus have adaptive abilities to colonize different environments. Their survival is ensured through efficient ways to detect cellulose in the environment and secrete various cellulases responsible for the degradation of insoluble substrates, transportation of soluble products via the cytoplasmic membrane, and assimilation of sugars. Furthermore, this 
fungus has the flexibility to respond to changes in the nutritional composition of the environment, and thus, is able to compete with other microorganisms [6]. Therefore, correct interpretation of environmental stimuli, using the available resources, ensures the success of this fungus in nature [7].

In response to an extracellular signal captured by a receptor, a signaling cascade is initiated, and this signal is transmitted via secondary messengers such as cyclic AMP (cAMP). The cAMP signaling pathway is a cascade that has crucial functions in all organisms and is highly conserved in fungi [7]. In fungi, this messenger is involved in stress response, sporulation, growth, virulence, mycoparasitism, carbon and lipid metabolism as well as other functions in response to extracellular signals [8-12]. It has been suggested that induction of cellulases in $T$. reese $i$ is related to a signaling pathway involving a cAMP-dependent protein kinase, and that, in certain circumstances the presence of this second messenger can cause an overproduction of some cellulases [7, 13]. However, the biosynthesis of these cellulases is not only induced by cellulose, but also by cellobiose, 1,5- $\delta$-lactone celobiono (an analog of cellobiose), lactose and sophorose. On the other hand, readily metabolizable carbon sources such as glucose, fructose, or glycerol repress expression of cellulases [14].

The regulation of transcription of cellulolytic complexes has been extensively studied, and some transcription factors involved in this process have already been identified [15]. However, little is known about how signals are transmitted to these transcription factors. True inductors, receptors, and pathways involved in the transduction of these signals to specific transcription factors have not been identified yet [16]. Many of these factors are induced under the conditions for which they are necessary, and they are degraded once they have done their function. Therefore, it is reasonable to assume that the activity of these transcription factors is also regulated by modifications that occur in response to changes in fungal culture conditions [7].

This study aimed to elucidate the involvement of cAMP in the expression of cellulase genes cel7a and cel6a in $T$. reesei using different inducers (i.e., cellulose, sophorose, lactose, and cellobiose), and a repressor (i.e., glucose). Our results suggest that cAMP controls the expression of cellobiohydrolases only in the presence of the inducer sophorose and is potentially involved in the secretion process of cellulases.

\section{Methods}

\section{Strains and growth conditions}

T. reesei QM9414 (ATCC 26921) and mutant $\Delta a c y 1$ [17] strains were obtained from the Institute of Molecular Biotechnology, Vienna, Austria. Strains were maintained in MEX medium [malt extract $3 \%(\mathrm{w} / \mathrm{v})$ and agar-agar $2 \%(\mathrm{w} / \mathrm{v})]$ at $4{ }^{\circ} \mathrm{C}$. QM9414 and $\Delta a c y 1$ were grown in
MEX medium at $28{ }^{\circ} \mathrm{C}$ for seven to 10 days to complete sporulation. For gene expression assays and cellulase activity measurements in QM9414, a suspension containing approximately $10^{8}$ spores $/ \mathrm{mL}$ was inoculated in $200 \mathrm{~mL}$ Mandels-Andreotti medium [18] containing $1 \%$ (w/v) cellulose (Avicel, Sigma, St. Louis, Missouri, EUA), or $1 \%(\mathrm{w} / \mathrm{v})$ lactose (Sigma, St. Louis, Missouri, EUA), or $1 \%(\mathrm{w} / \mathrm{v})$ cellobiose (Serva, Heidelberg, Germany), or $2 \%(\mathrm{w} / \mathrm{v})$ glucose (Sigma, St. Louis, Missouri, EUA), or in $20 \mathrm{~mL}$ of the same medium containing $1 \mathrm{mM}$ sophorose (Serva, Heidelberg, Germany) as the sole carbon source. For gene expression assays in $\Delta a c y 1$, a suspension containing approximately $10^{8}$ spores $/ \mathrm{mL}$ was inoculated in $200 \mathrm{~mL}$ Mandels-Andreotti medium containing $1 \%$ $(\mathrm{w} / \mathrm{v})$ cellulose (Avicel), or in $20 \mathrm{~mL}$ of the same medium containing $1 \mathrm{mM}$ sophorose as the sole carbon source, with or without $1 \mathrm{mM}$ dibutyryl-cAMP (dbcAMP - Sigma, St. Louis, Missouri, EUA). Cultures were incubated on an orbital shaker $(200 \mathrm{rpm})$ at $28{ }^{\circ} \mathrm{C}$ for 24,48 , and $72 \mathrm{~h}$ using cellulose, lactose, or cellobiose; for 24 and $48 \mathrm{~h}$ using glucose; and 2, 4 and $6 \mathrm{~h}$, or 6, 12 and $18 \mathrm{~h}$ using sophorose. For the latter, the mycelium was previously grown in $1 \%$ (w/v) glycerol (Sigma, St. Louis, Missouri, EUA) for $24 \mathrm{~h}$. After incubation, the mycelia were washed with Mandels-Andreotti medium without peptone, and transferred to $20 \mathrm{~mL}$ of Mandels-Andreotti medium without peptone containing $1 \mathrm{mM}$ sophorose. All experiments were performed in three biological replicates. The resulting mycelia and supernatants were collected by filtration, frozen and stored at $-80{ }^{\circ} \mathrm{C}$ until RNA and protein extraction, as well as cellulose activity measurements were performed.

\section{Determination of cellulase activity}

Determination of cellulase activity was performed using Cellulose Azure $^{\oplus}$ (Sigma, St. Louis, Missouri, EUA) as the substrate. This methodology involves the release of a blue color when cellulases are present. In these experiments, the reaction mixture consisted of Cellulose Azure, $100 \mathrm{mM}$ sodium citrate buffer $\mathrm{pH}$ 5.0, and samples from culture supernatants. Reactions were performed at $55^{\circ} \mathrm{C}$ for $30 \mathrm{~min}$. After this, the alcohol precipitation reagent (APR) was added according to the manufacturer instructions, and samples were centrifuged. Supernatant was collected, and samples were measured using a spectrophotometer (Spectrophotometer $\mathrm{xMark}^{\mathrm{TM}}$ Micro, Bio-Rad, San Francisco, CA, USA) at $575 \mathrm{~nm}$. A calibration curve was created using varying concentrations of the substrate incubated at $50{ }^{\circ} \mathrm{C}$ for $4 \mathrm{~h}$ in $100 \mathrm{mM}$ sodium citrate buffer $\mathrm{pH} 5.0$ and $15 \mathrm{U} / \mathrm{mL}$ cellulase purified from Trichoderma reesei in the Microbiology Laboratory of Cell Biology at the Biology Department (Faculty of Philosophy, Sciences, and Letters of Ribeirao Preto, University of Sao Paulo, Brazil). Activity was expressed 
as the ratio between change in growth factor absorbance and the absorbance obtained in the standard curve, per min, and per amount of enzyme added to the reaction.

\section{RNA extraction}

Total RNA was extracted from mycelia of each sample using TRIzol ${ }^{\circ}$ RNA kit (Invitrogen Life Technologies, Carlsbad, CA, USA), according to the manufacturer instructions. RNA concentration was determined using spectrophotometric optical density at $260 / 280 \mathrm{~nm}$, and RNA integrity was verified by electrophoresis in $1 \%$ agarose gels.

\section{Quantitative real time-PCR analysis}

Approximately $1 \mu \mathrm{g}$ RNA was treated with DNAseI (Fermentas, Waltham, Massachusetts, USA) and reversetranscribed to cDNA using the First Strand cDNA kit Maxima ${ }^{\text {Tw }}$ Synthesis (Thermo Scientific, Waltham, Massachusetts, USA) according to manufacturer instructions. cDNA was diluted 1:50 for real-time PCR analysis in a Bio-Rad CFX96 ${ }^{\text {tm }}$ System (Bio-Rad, San Francisco, CA, USA), using SsoFast ${ }^{\mathrm{Tm}}$ EvaGreen $^{\circ}$ Supermix (Bio-Rad, San Francisco, CA, USA) for signal detection, in accordance with the manufacturer instructions. Primers used in this study are listed in Table 1 . The gene encoding actin was used as a constitutive expression control [19]. The following amplification reaction was used: $95{ }^{\circ} \mathrm{C}$ for $10 \mathrm{~min}$ followed by 39 cycles at $95{ }^{\circ} \mathrm{C}$ for $10 \mathrm{~s}, 60{ }^{\circ} \mathrm{C}$ for $30 \mathrm{~s}$ followed by a dissociation curve at 60 to $95^{\circ} \mathrm{C}$ with an increment of $0.5{ }^{\circ} \mathrm{C}$ every $10 \mathrm{~s}$. Gene expression values were calculated according to the $2^{-\triangle \Delta C T}$ method, using the QM9414 strain grown in glucose as the reference sample [19]. Data analysis was performed using GraphPad Prism v5.1 software.

\section{Extraction and measurement of cAMP}

After fungal growth in Mandels-Andreotti medium, mycelia were collected and frozen in liquid nitrogen. For measurement of cAMP, mycelia were macerated, and samples were transferred to polypropylene tubes and weighed. Then, 10 volumes of $0.1 \mathrm{M} \mathrm{HCl}$ were added, and the tubes were centrifuged at $6,000 \times g$ for $10 \mathrm{~min}$ at $4{ }^{\circ} \mathrm{C}$. The supernatant was used directly for measurement of cAMP levels using the "Direct cAMP Enzyme

Table 1 Primers used in this study

\begin{tabular}{llll}
\hline No. & Code & Protein ID & $5^{\prime} \rightarrow 3^{\prime}$ \\
\hline 1 & Cel6a F & 72567 & ACA AGA ATG CAT CGT CTC CG \\
2 & Cel6a R & & TGT TCC ACC CGT TGT AGT TG \\
3 & Cel7a F & 123989 & CCG AGC TTG GTA GTT ACT CTG \\
4 & Cel7a R & & GGT AGC CT CTT GAC TGA GT \\
5 & Actin F & 44504 & TGA GAG CGG TGG TAT CCA CG \\
6 & Actin R & & GGT ACC ACC AGA CAT GAC AAT GTT \\
\hline
\end{tabular}

Immunoassay" kit (Sigma, St. Louis, Missouri, EUA) according to the manufacturer instructions. The content of intracellular cAMP was relative to protein concentration in the same sample.

\section{Protein extraction and western blot}

Secreted proteins from QM9414 and $\Delta a c y 1$ strains were precipitated using $10 \%$ tricarboxylic acid (TCA) in acetone, and allowed to stand at $-20{ }^{\circ} \mathrm{C}$ overnight. Samples were centrifuged at $10,000 \times g$ for $10 \mathrm{~min}$ at $4{ }^{\circ} \mathrm{C}$, and the supernatant was removed. $\beta$-mercaptoethanol $(0.07 \%)$ in acetone was added, and samples were centrifuged at $10,000 \times g$ for $10 \mathrm{~min}$ at $4{ }^{\circ} \mathrm{C}$. This process was repeated three times, discarding the supernatant after each centrifugation. Then, samples were resuspended in buffer (25 mM Tris pH 6.8, $12.5 \%$ SDS, $50 \%$ glycerol and $0.05 \%$ bromophenol blue) and heated for $5 \mathrm{~min}$ at $100{ }^{\circ} \mathrm{C}$. For intracellular protein extraction [20], mycelia grown in sophorose were used as the source [21]. Total protein concentration was determined according to the Bradford method using the Coomassie Plus Protein Assay Reagent (Thermo Scientific, Waltham, Massachusetts, USA) at $595 \mathrm{~nm}$, in a spectrophotometer (Spectrophotometer xMark ${ }^{\mathrm{m}}$ Micro, Bio-Rad). Subsequently, $12 \mu \mathrm{g}$ of protein samples (secreted and intracellular proteins) were subjected to electrophoresis on $10 \%$ polyacrylamide gels. After electrophoresis, proteins were transferred to nitrocellulose membranes (GE Healthcare) for $40 \mathrm{~min}$ using a humid system (Trans-Aid ${ }^{\mathrm{m}}$ Blot ${ }^{\circ}$ Turbo transfer system, Bio-Rad) with transfer buffer ( $25 \mathrm{mM}$ Tris, $197 \mathrm{mM}$ glycine, 20 \% methanol). Membranes were blocked for $1 \mathrm{~h}$ at room temperature in tris-buffered saline (TBS) containing $0.05 \%$ Tween (TBS-Tween) and $5 \%$ skimmed milk. Membranes were then incubated overnight at $4{ }^{\circ} \mathrm{C}$ with a rabbit polyclonal anti-Trichoderma viride cellulase (CEL7A) antibody (MyBioSource) diluted 1:1000. Membranes were then washed three times $10 \mathrm{~min}$ each with TBS-Tween and incubated for $1 \mathrm{~h}$ with the corresponding peroxidase-conjugated anti-rabbit secondary antibody (Invitrogen). Membranes were again washed three times $10 \mathrm{~min}$ each with TBS-Tween and developed using Enhanced Chemiluminescence reagent (ECL, GE Healthcare) according to the manufacturer instructions. Finally, ECL membranes were photographed using $\mathrm{ChemiDoc}^{\mathrm{Tm}} \mathrm{XRS}+$ (BioRad) photo-documentation system.

\section{Indirect immunofluorescence and confocal microscopy}

Aliquots withdrawn from $\Delta a c y 1$ at 6,12 , and 18 h during culture were centrifuged at $6,000 \times g$ and washed with $0.1 \mathrm{M}$ phosphate buffered saline (PBS) at $\mathrm{pH}$ 7.4. After washing, mycelia were immersed in $2 \%$ paraformaldehyde in PBS at room temperature and embedded in optimal cutting temperature (OCT) compound (Tissue Tek, Sakura Finetec, Torrence CA) in a suitable tissue mold for 
freezing. Subsequently the inclusion, mycelia were sectioned on a cryostat (10 $\mu \mathrm{m}$ thick), and sections were mounted on silanized slides kept under vacuum for $12 \mathrm{~h}$ to affix the sections. Slides were then stored at $-20^{\circ} \mathrm{C}$. For confocal microscopy, slides were washed with PBS and incubated with a $3 \%$ bovine serum albumin (BSA) solution for $1 \mathrm{~h}$ at room temperature to block non-specific binding. Then, slides were incubated for $1 \mathrm{~h}$ at room temperature with a rabbit polyclonal anti-Trichoderma viride cellulase (CEL7A) antibody (MyBioSource) diluted 1:1000 in $3 \%$ BSA solution in PBS. Slides were then incubated with an Alexa Fluor 488-conjugated secondary antibody (Molecular Probes, Life Technologies) diluted 1:5000 in PBS with $3 \%$ BSA at room temperature. Slides were washed with PBS between steps and images were acquired using a Leica TCS SP5 confocal microscope.

\section{Analytical methods}

Biomass was determined by gravimetric analysis for glycerol culture. After $24 \mathrm{~h}$ of culture, mycelia was filtrated on filter paper and incubated at $70{ }^{\circ} \mathrm{C}$ for $3 \mathrm{~h}$ and then weighed. For cellulose culture, biomass was indirectly measured by the amount of intracellular protein quantified by the Quick Start Bradford protein assay kit (Bio-Rad) with bovine serum albumin (BSA) as a standard.

\section{Results}

Analysis of the expression of the cellobiohydrolases cel7a and cel6a and cellulase activity using different carbon sources

To guarantee comparisons between strains, we first examined the growth pattern of strains on cellulose and glycerol. No significant difference in growth between Sacy1 mutant strain and the parental QM9414 as in cellulose as in glycerol was observed [see Additional file 1]. Expression of the two most expressed cellulase genes in T. reesei (cel7a and cel6a) was analyzed after growing the QM9414 strain in different carbon sources, as described in the Methods section. As shown in Fig. 1, cel7a and cel6a expression increased when the fungus was grown in cellulose, sophorose, or lactose. Using cellulose, cel7a expression increased 10-fold at 48 and $72 \mathrm{~h}$ (Fig. 1a). Regarding sophorose and lactose, the highest cel7a expression (30-fold) was observed after 6 and $48 \mathrm{~h}$, respectively. The expression profile of cellobiohydrolase cel6a was similar to that of cel7a, with the highest expression levels in sophorose at $6 \mathrm{~h}$ (80-fold) and lactose at $48 \mathrm{~h}$ (90-fold) (Fig. 1b). As expected, neither cel7a nor cel6a showed detectable expression levels in glucose. Interestingly, no expression of cel7a and cel6a was observed in cellobiose.

Similar to qPCR-RT results, the QM9414 strain exhibited higher cellulolytic activity in cultures with cellulose, sophorose, and lactose (Fig. 2). However, different to

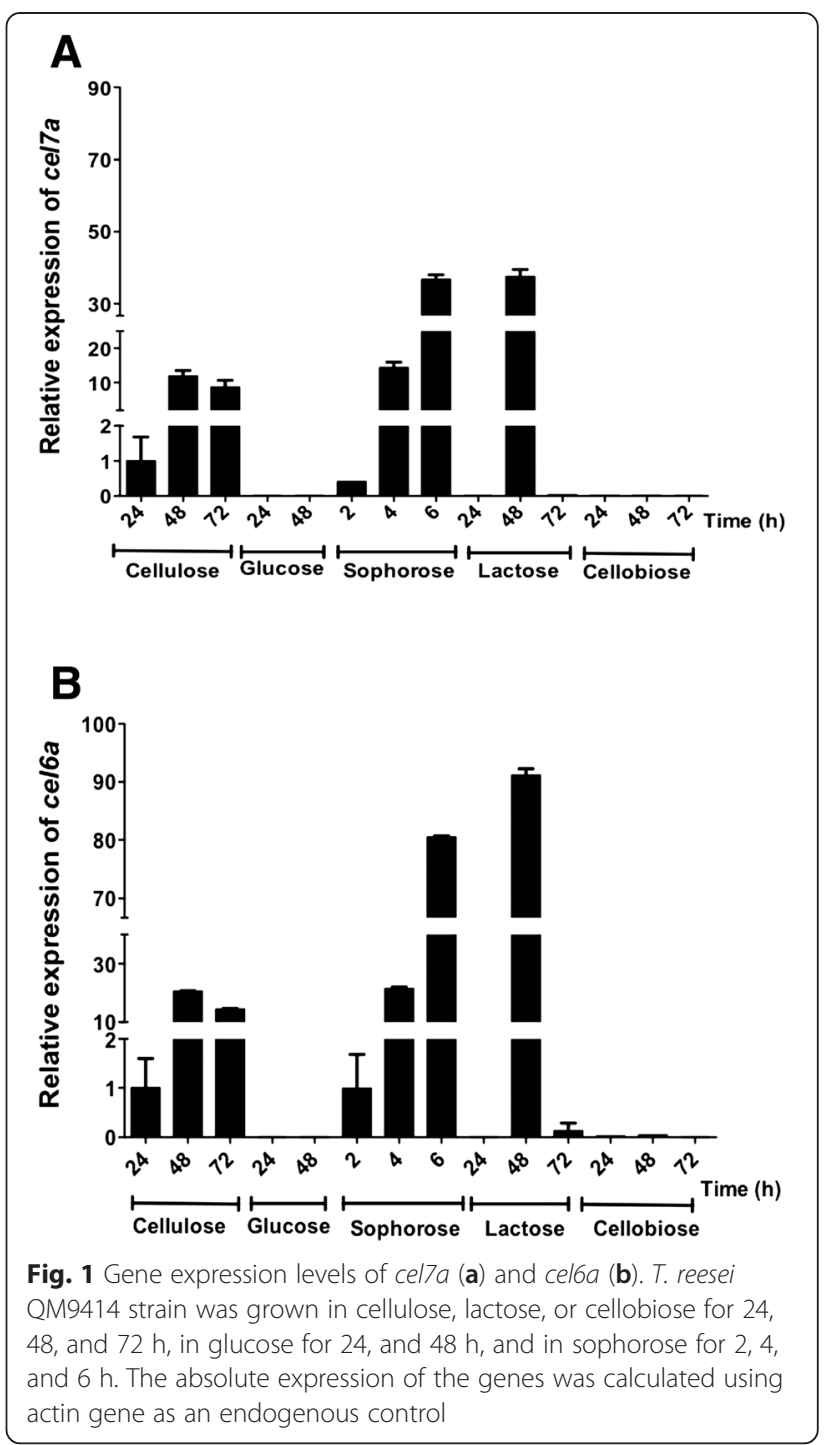

what was observed in the expression analysis, which showed an increase of cel7a and cel6a expression after $6 \mathrm{~h}$, cellulolytic activity in sophorose increased mainly at $2 \mathrm{~h}$ in culture $(24.5 \mathrm{U} / \mathrm{mL})$. A similar pattern was observed growing QM9414 in lactose, where cellulolytic activity reached high levels at $72 \mathrm{~h}(22.5 \mathrm{U} / \mathrm{mL})$. Cellulolytic activity in cellulose was higher at 48 and $72 \mathrm{~h}(24.5$ $\mathrm{U} / \mathrm{mL}$ and $20.7 \mathrm{U} / \mathrm{mL}$, respectively). On the contrary, hydrolytic activity in glucose was lower than in other carbon sources, being 6, 5, and 4.8 times lower than in cellulose, sophorose, lactose and cellobiose, respectively. Interestingly, our results showed no correlation between the expression profile and cellulolytic activity in cellobiose. The cellulolytic activity showed a steady increase from $24 \mathrm{~h}$ and a maximum at $72 \mathrm{~h}(19.8 \mathrm{U} / \mathrm{mL})$ (Fig. 2). This result may be explained from the fact that the azure-Cellulose ${ }^{\bullet}$ method detects total cellulolytic activity without distinguishing specific cellulases. 


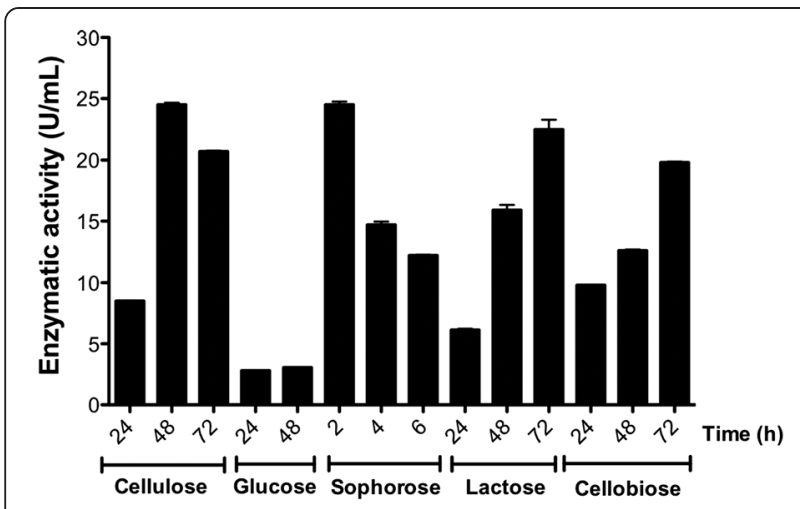

Fig. 2 Total cellulolytic activity in different carbon sources. T. reesei QM9414 strain was grown in cellulose, lactose, or cellobiose for 24 48, and $72 \mathrm{~h}$, in glucose for 24, and $48 \mathrm{~h}$, and in sophorose for 2, 4, and $6 \mathrm{~h}$. Activity was measured using the azure-Cellulose method and is expressed as the ratio between growth factor and the absorbance obtained in the standard curve equation, per minute, and per amount of enzyme added to the reaction

Intracellular CAMP levels are regulated according to the carbon source

We found that cellobiohydrolase gene expression and cellulolytic activity profile varied depending on the carbon source used in the experiments of induction. Previous studies have suggested that intracellular levels of cAMP may regulate both expression of cellulase genes and activity of the enzyme. To address this, we measured intracellular levels of cAMP in mycelia from the QM9414 strain grown in cellulose, sophorose, lactose, cellobiose, and glucose.

Our results showed that intracellular levels of cAMP were modulated in a carbon source-dependent manner. As shown in Fig. 3, cAMP concentration (229.8 pmol/mg) at $4 \mathrm{~h}$ was at least 4 times higher in the presence of sophorose than in the presence of other carbon sources. In cellulose, the highest cAMP concentration was quantified after $24 \mathrm{~h}$ cultivation $(61.7 \mathrm{pmol} / \mathrm{mg})$. This value was 3 times higher than the maximum levels found in glucose

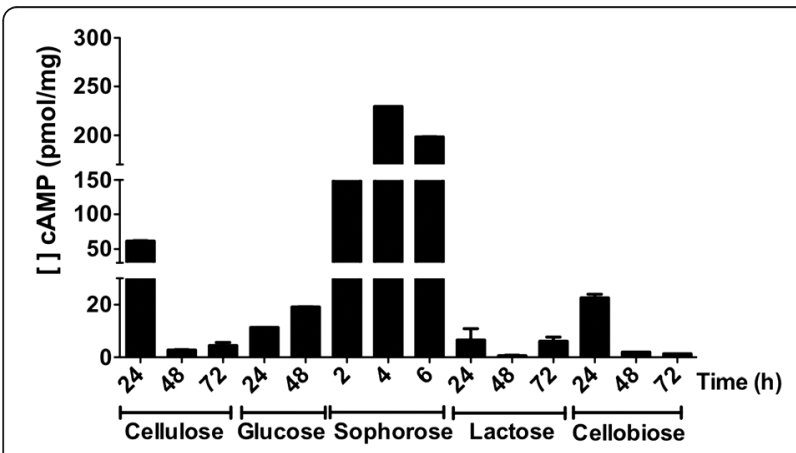

Fig. 3 Intracellular CAMP content. T. reesei QM9414 strain was grown in cellulose, lactose, or cellobiose for 24,48 , and $72 \mathrm{~h}$, in glucose for 24, and $48 \mathrm{~h}$, and in sophorose for 2, 4, and $6 \mathrm{~h}$
(19.13 $\mathrm{pmol} / \mathrm{mg})$ at $48 \mathrm{~h}$ and 2.5 times higher than with cellobiose (22.5 pmol/mg) at $24 \mathrm{~h}$ (Fig. 3). Concomitantly, lower levels of cAMP were detected in lactose and cellulose at 48 and $72 \mathrm{~h}$, and cellobiose at 48 and $72 \mathrm{~h}$. Altogether, our findings showed that in a similar manner to cellobiohydrolase expression and cellulolytic activity, intracellular levels of cAMP are also dependent on the inducer. This suggests a regulatory role of cAMP in the control of cellulase expression only in the presence of sophorose, since expression levels of cel7a and cel6a were increased in both lactose and cellulose. On the other hand, intracellular concentration of cAMP did not exhibit the same profile regarding carbon sources.

\section{Analysis cel7a and cel6a expression in the $\Delta$ acy 1 mutant strain}

The expression profile of the two most abundant cellobiohydrolases in the parental lineage QM9414 was directly regulated in response to four different inducers. Here we showed that the increased expression of cel7a and cel6a correlates with high intracellular levels of cAMP in the presence of sophorose. Then, we wanted to assess how cAMP regulates cellulase expression in $T$. reese $i$, and whether the effects of this secondary messenger are determined by the carbon source. To assess this, we used the $\Delta a c y 1$ mutant strain, which features a deletion in the adenylate cyclase gene, and dbcAMP (dibutyryl-cAMP) in the induction medium. Growth of the $\Delta a c y 1$ mutant strain was assessed in cellulose and sophorose at 72 and $6 \mathrm{~h}$, respectively, which were the time points and carbon sources that showed the highest expression of cellobiohydrolases.

Figure 4 shows that in the presence of sophorose and dbcAMP, expression of the cellobiohydrolases cel7a (Fig. 4a) and cel6a (Fig. 4b) was significantly increased relative to cellulose, either in the presence or absence of dbcAMP. Interestingly, dbcAMP effects were mainly evident in the presence of the most potent inducer of cellulase expression (i.e., sophorose) than in cellulose at the same concentration. As a result of the abolition of adenylate cyclase expression in the $\Delta a c y 1$ mutant strain, the only source of cAMP in the induction medium was dbcAMP. Therefore, supplementation with exogenous cAMP enhanced the expression of cellobiohydrolases in the $\Delta a c y 1$ strain, suggesting an essential effect of cAMP in the regulation of cellulase gene expression in T. reesei.

\section{Detection of total cellulase in mycelia and secretome of $\Delta$ acy1 and QM9414}

The cAMP signal transduction pathway controls a wide variety of processes in fungi. In the $\Delta a c y 1$ mutant strain processes such as carbon metabolism, conidiation, mating, phototropism, and synthesis and secretion of proteins may be disturbed because of adenylate cyclase 


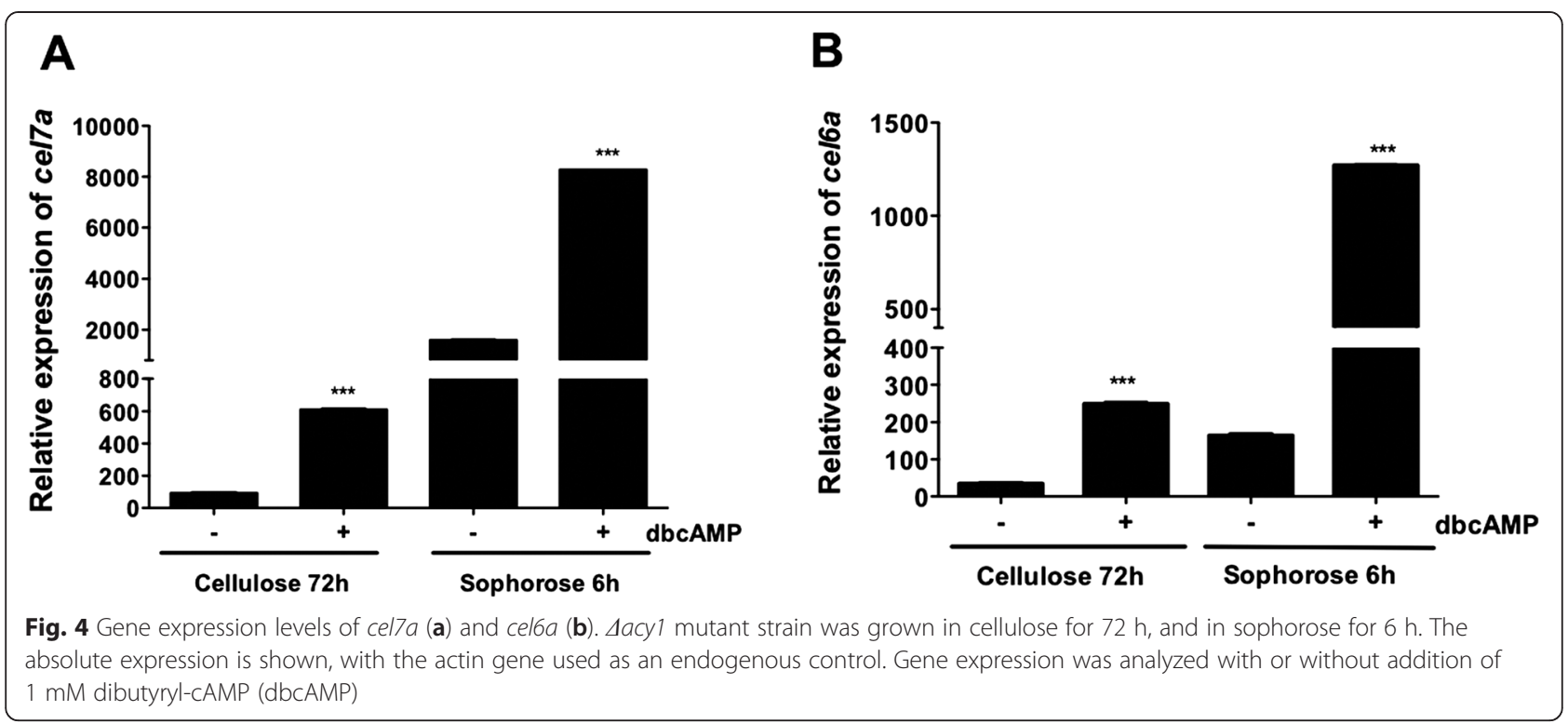

deletion. Using western blot and indirect immunofluorescence microscopy approaches we assessed cellulase content in the mycelia (intracellular protein) and in the secretome of QM9414 and $\Delta a c y 1$ strains.

Our immunofluorescence results revealed that dbcAMP strongly influenced cellulase expression in the $\triangle a c y 1$ mutant strain (Fig. 5). Addition of dbcAMP promoted an increase in cellulase expression at $6 \mathrm{~h}$ in induction medium with sophorose. Similar results were also observed at 12 and $18 \mathrm{~h}$ (Data not shown), with the same increase in cellulase expression. We observed an accumulation of cellulases in the hyphal tip (Fig. 5, dashed squares), when dbcAMP was added. These results, together with the qRT-PCR and activity analyses, suggest that dbcAMP directly controls expression and secretion of cellulase in $T$. reesei.

We found no correlation between expression level of cellobiohydrolases and activity in at $6 \mathrm{~h}$ of incubation in the presence of sophorose. On the other hand, the immunofluorescence analysis showed that at $6 \mathrm{~h}$ in the presence of dbcAMP there are increased of cellulase expression. So, we asked if occurs a retention of cellulases in the mycelium. Our western blotting results showed a detection of an approximately band of 59-68 kDa (relative to CEL7A), and we observed a lower content of CEL7A in the secretome of $\triangle a c y 1$ strain in the absence of dbcAMP (Fig. 6a, upper panel). The addition of dbcAMPC increased the content of CEL7A in the secretome. The differences were more evident at $12 \mathrm{~h}$ and $18 \mathrm{~h}$, being the higher expression of CEL7A reached always in the presence of dbcAMP. The detection of CEL7A in the mycelium of $\triangle a c y 1$ (Fig. 6b, upper panel) revealed an elevated content of this cellulase at $6 \mathrm{~h}$ in the absence of dbcAMP, justifying the lower detection in the secretome. In the same way, the addition of dbcAMP at $6 \mathrm{~h}$ induced a high secretion of CEL7A as demonstrated by the decreased of protein in the mycelium (Fig. 6b, upper panel). In addition, we observed that the content of CEL7A in the mycelium at $12 \mathrm{~h}$ and $18 \mathrm{~h}$ were higher than at $6 \mathrm{~h}$, but the content of protein in the secretome remain increased at $12 \mathrm{~h}$ and $18 \mathrm{~h}$ relative to mycelium. In the $\Delta a c y 1$ strain the expression of cellulase was higher after $6 \mathrm{~h}$ of cultivation, being the content of CEL7A maximum at $18 \mathrm{~h}$. Oppositely, in the secretome of QM9414, the maximum content of CEL7A was detectable at $12 \mathrm{~h}$ in the absence of dbcAMP (Fig. 6a, lower panel). Moreover, we demonstrated a lower content of cellulase in the mycelium of QM9414 at $6 \mathrm{~h}$, $12 \mathrm{~h}$ and $18 \mathrm{~h}$ compare to $\Delta a c y 1$ mutant strain (Fig. 6b). So, the analysis of secretome and mycelium protein content revealed that addition of dbcAMP alters the pattern of CEL7A secretion in T. reesei. The content of CEL7A in the presence of dbcAMP had no change in the secretome, but comparing the protein content in the mycelium in the absence and in the presence of dbcAMP, we observed the accumulation of protein in the mycelium after addition of dbcAMP mainly at $6 \mathrm{~h}$ and $12 \mathrm{~h}$ (Fig. 6b, lower panel). Our findings suggest that cAMP may regulate the expression, and secretion of cellobiohydrolases in $T$. reesei in the presence of the inducer sophorose.

\section{Discussion}

In $T$. reesei, it is well established that expression of cellulases is carbon source-dependent [12]. Our group has described the influence of different carbon sources in the regulation of cellulase gene expression $[22,4]$. Although various studies have discussed that cellulase genes are dependent on induction, little is 

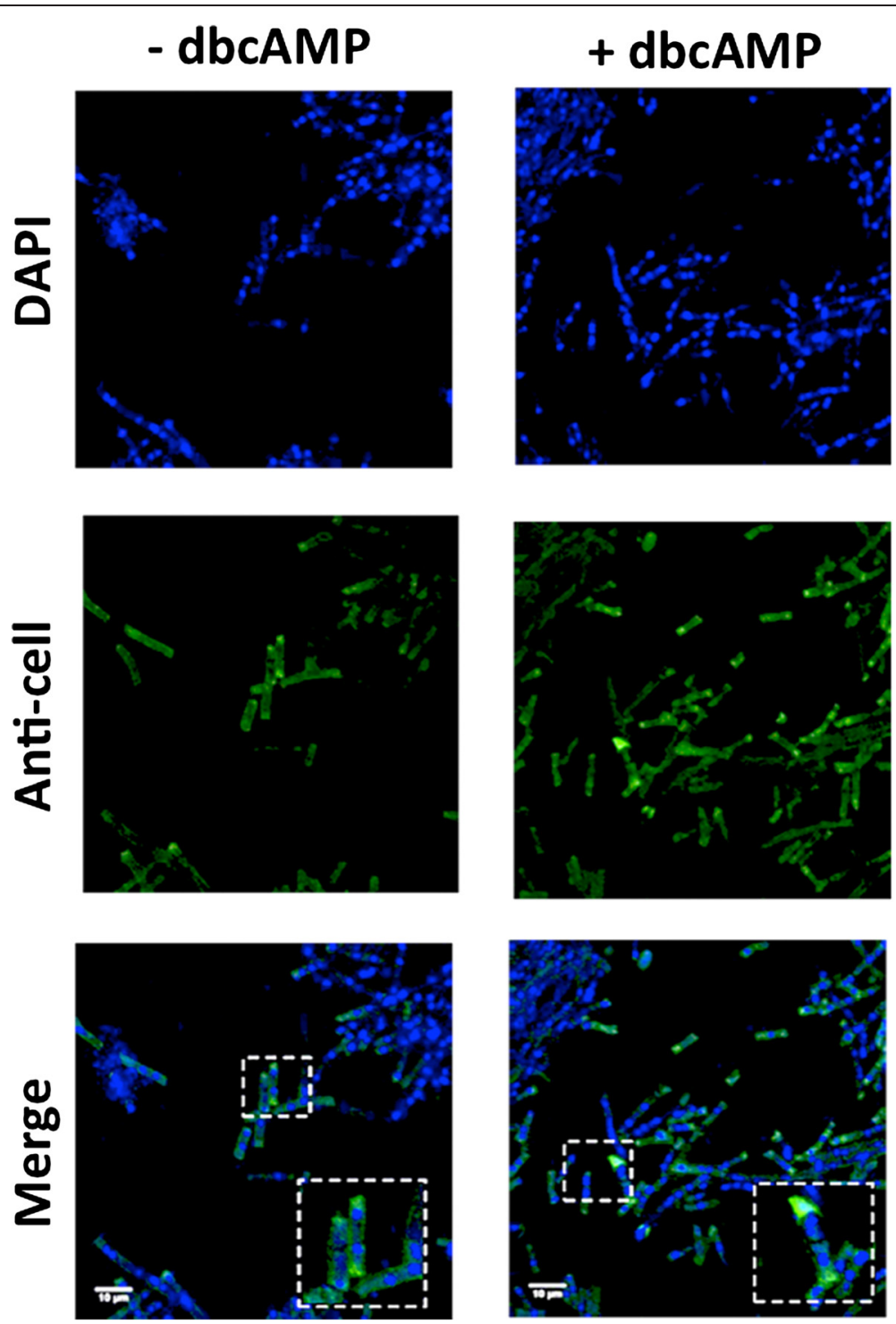

Fig. 5 Cellulase detection using indirect immunofluorescence in the $\Delta a c y 1$ mutant strain. The fungus was grown in sophorose for $6 \mathrm{~h}$ in the presence or absence of dibutyryl-cAMP (dbcAMP). To detect cellulase in the mycelia we used a rabbit polyclonal anti-Trichoderma viride cellulose (CEL7A) antibody diluted 1:1000, and an Alexa Fluor 488-conjgated secondary antibody diluted 1:5000. Furthermore, the fluorochrome DAPI was used to detect cell nuclei. Images were acquired on a Leica TCS SP5 confocal microscope. Lens 40x, and zoom 3.8

known about the nature of the inducer, and the signaling pathways controlling cellulase gene expression in this fungus [16].

In T. reesei the only well characterized signal transduction cascades are: a light-modulated cellulase production (G protein-mediated, Gna1 and Gna3), a PAS/LOV domain protein ENVOY and cAMP-dependent protein kinase A signaling $[7,17,18,23]$, regulation of sexual development [24], and cellulose- and cAMP-independent modulation of cellulase production mediated by Ras GTPase [25]. Recently, Wang et al. [26, 27] described the role of MAPK signaling in the regulation of both expression and activity of cellulases in $T$. reesei.
The cyclic AMP (cAMP) pathway is a central signaling cascade with crucial functions in all organisms. In Saccharomyces cerevisiae, nutrient sensing and pseudohyphal differentiation in response to nitrogen-limiting conditions is controlled by this pathway [28-30]. In the fission yeast, Schizosaccharomyces pombe, cAMP mediates the effect of glucose and gluconeogenesis in spore germination, and regulates mating in response to either glucose or nitrogen deprivation [31, 32]. In filamentous fungi such as Neurospora crassa and Aspergillus species, cAMP controls hyphal growth polarity and morphogenesis, conidiation, and spore germination [33-36]. Furthermore, as a secondary messenger, cAMP is involved 

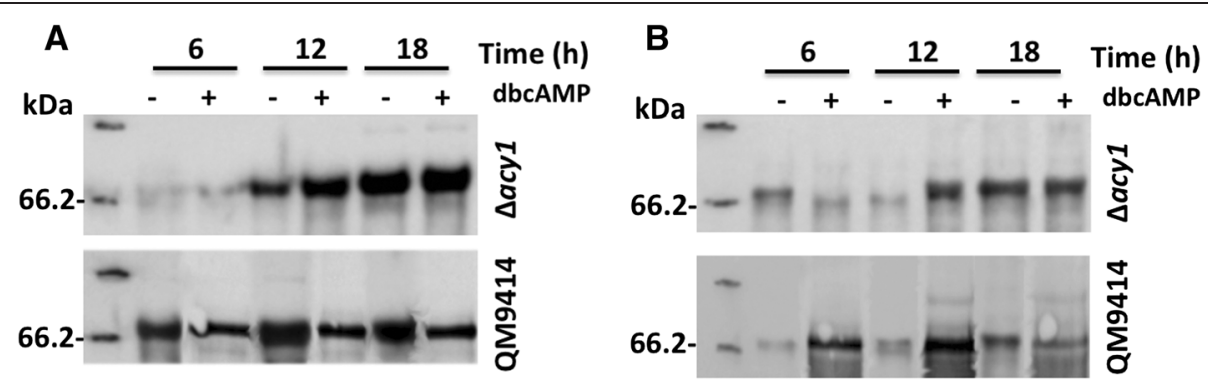

Fig. 6 Analysis of cellulase expression in $\triangle a c y 1$ and QM9414 strains. Fungi were grown in sophorose for 6, 12, and $18 \mathrm{~h}$ in the presence or absence of dibutyryl-cAMP (dbcAMP). a Expression of CEL7A in the secretome of the $\triangle$ acy 1 mutant strain (upper panel) and QM9414 parental strain (lower panel) in the presence or absence of dbcAMP. b Expression of CEL7A in the mycelium of the $\triangle a c y 1$ mutant strain (upper panel) and QM9414 parental strain (lower panel) in the presence or absence of dbcAMP

in stress response, carbon and lipid metabolism, sporulation, development, virulence, mating, mycoparasitism, and other responses to extracellular signals [8-12, 37].

Previous studies in $T$. reese $i$ have suggested that cAMP signaling regulates cellulase gene expression [13]. Sestak and Farkas (1993) [13] showed that addition of cAMP can double the efficacy of sophorose in the induction of endoglucanase formation. In another filamentous fungus, Cryptococcus albidus, cAMP was shown to be important for xylanase production [38]. According to Tisch and Schmoll [7], the most important role of cAMP is to activate cAMP-dependent protein kinase A (PKA), which in turn initiates a phosphorylation cascade and activates/inactivates further target genes. Schuster et al. (2012) [17] showed that both adenylate cyclase (ACY1) and PKA (catalytic subunit, PKAC1) were important factors in cellulase gene expression in T. reesei. However, the mechanisms related to the impact on cellulase gene expression remain unclear.

In the present study, we showed that the effect of cAMP in the regulation of cellulase gene expression in T. reesei was carbon source-dependent and that the role of this secondary messenger is more evident in the presence of sophorose. This sugar is formed by transglycosylation of cellobiose during cellulose hydrolysis [39], and it is thought to be the natural inducer of cellulase formation, being its presence in culture fluids of $T$. reesei commonly described [40-42]. Moreover, this disaccharide has a dual role in $T$. reesei in $\beta$-glucosidase repression and cellulase induction [16]. The efficiency of sophorose and other disaccharides such as lactose in the induction of cellulase has also been observed in Trichoderma viride, Pseudomonas fluorescens var. cellulosa, Acremonium cellulolyticus, Penicillium echinulatum [41, 43-45]. Interestingly, our results showed a low expression of cellulase genes in the presence of cellobiose. This may be explained by the fast hydrolysis of this sugar by $\beta$-glucosidase activity, which converts cellobiose to glucose, a carbon source repressor of cellulase synthesis [46, 47]. Altogether, our results suggest an intriguing mechanism by which the fungus identifies the available carbon source in the culture medium and controls the full transcription machinery of genes responsible for carbon source metabolism. In addition, our results highlight the role of cAMP as a sophorose sensor.

Our qRT-PCR results showed that expression of cellobiohydrolases cel7a and cel6a increased in the presence of cellulose, sophorose, and lactose. However, in the presence of sophorose, addition of dbcAMP induced an increase of 5- and 7.8-fold in the expression of cel7a and cel6a, respectively. As observed, the expression of cel6a was more sensitive to intracellular levels of cAMP than cel7a. Interestingly, the high expression levels of cel7a and cel6a correlated with high intracellular cAMP levels. The influence of cAMP in the regulation of cellulase expression has been shown in important fungi such as Trichoderma, Penicillium, and Aspergillus spp., which have biotechnological interest [41, 45]. Furthermore, $\mathrm{Hu}$ et al. [48], in a study in Penicillium decumbens, showed the effect of PGA3, a group III G-protein $\alpha$ subunit, on the expression of amylases and cellulases. Deletion of pga3 resulted in impaired amylase production, and significantly decreased transcription of the major amylase gene amy15A. Moreover, supplementation with exogenous cAMP or its analog dbcAMP restored amylase production in the $\triangle p g a 3$ strain, suggesting an essential role for PGA3 in amylase synthesis by controlling cAMP levels. Conversely, transcription of the cellulase gene cel7A-2 increased in the $\triangle p g a 3$ strain, although cellulase activity in the medium was not affected. Dong et al. [49] showed that exogenous cAMP could increase cellulase synthesis under derepression conditions. Nevertheless, cAMP has an ambiguous role in the regulation of cellulase expression because at lower concentrations increases cellulolytic activity, while high levels of cAMP repress cellulase synthesis. Interestingly, Herrera-Herrera et al. [50] showed that in Cellulomonas flavigena, an actinobacteria with special interest for its ability to degrade cellulose and 
hemicellulose, addition of exogenous cAMP in repressor culture medium decreased catabolite repression, while supplementation enhanced cellobiohydrolase production. Similarly, Rizzati et al. [51] showed in Aspergillus phoenicis that repression of xylanase expression by glucose was partially reversed by addition of cAMP or dbcAMP.

In our experiments using cellulose, we showed that expression of cel7a and cel6a in the $\Delta a c y 1$ strain were 6- and 7.3-fold higher with addition of dbcAMP. This increase may be explained because degradation of cellulose releases inducer disaccharides such as sophorose, which in turn may active the expression of cellulolytic enzymes. This result corroborates our hypothesis that cAMP affects the expression of cellobiohydrolase, and that this regulation seems to be dependent on the type of carbon source, since changes in transcription of related genes were observed in both cellulose and sophorose, using the same concentration of dbcAMP. Hu et al. [48] described a different regulation in $P$. decumbens, in which expression of amylases and cellulases seemed to be independent of the carbon source. Taken together, our results suggest that the signaling pathway in response to sophorose involves a cAMP-dependent protein kinase that may control the expression of cellulolytic enzymes in the presence of easily metabolizable disaccharides, and that is also involved in the early stage of carbon sensing.

Our results revealed that the $\Delta a c y 1$ mutant strain showed a high expression of cellobiohydrolases in the presence of both cellulose and sophorose. As discussed above, this expression increased with supplementation of exogenous cAMP. This finding suggests that cAMP is not essential for cellulase expression, and that alternative pathways may interact with cAMP-dependent signaling to control expression of cellulolytic enzymes. In this regard, Schmoll et al. [18] showed that in T. reesei intracellular cAMP levels were positively correlated with cellulase expression in the presence of light. Furthermore, sensing of environmental signals mediated by Gprotein coupled receptors (GNA1 and GNA3) modulates considerably cellulase transcript levels, and the extent of this adjustment is dependent on light status. The critical light regulator ENVOY in T. reesei [52], is a small protein that contains a single PAS/LOV domain. The expression of the env1 gene is very low in darkness, but upon illumination the abundance of its transcript increases up to 500-fold. Recently, Tisch et al. [7] reported that ENVOY is involved in signal transduction via Gproteins, acting positively in the feedback of gnal and in the cAMP/protein kinase A pathway, controlling the function of the corresponding phosphodiesterase, although the mechanism is still unclear. In T. atroviride, a GPCR (GPR-1) that activates heterotrimeric G-proteins senses different carbon sources, and the $G \alpha$ proteins (tga1 or tga3) can activate adenylate cyclase that in turn may control intracellular levels of cAMP [53]. Thus, our results obtained in the $\triangle a c y 1$ strain provide evidence of the existence of a cAMP-independent pathway that controls cellulase expression, and may involve a receptor that senses different carbon sources; the effects of this sensing are more evident in the presence of disaccharides such as sophorose. These results are also in agreement with other studies $[13,54,55]$, which showed that cAMP may be an important signal for the regulation of cellulase formation, but not the only one.

The effect of cAMP on the regulation of cellulase expression in T. reesei was also revealed in our comparative analysis of immunofluorescence and western blot. Immunofluorescence data clearly showed that supplementation with exogenous cAMP controls expression of cel7a in sophorose induction. In addition, we observed that in the $\triangle a c y 1$ mutant strain there is a delay in the secretion of cellobiohydrolase CEL7A compared to the parental QM9414. Kinetic studies have shown that secretion is not faster in $T$. reesei than in other species. However, a good secretion capability depends on the capacity of the secretion machinery [56]. Altogether, our results suggest an involvement of intracellular cAMP levels in the regulation of cellulase secretion.

Filamentous fungi such as $T$. reesei and $A$. niger, produce large amounts of extracellular cellulolytic enzymes, while some strains mostly produce them in a multienzyme complex called cellulosome, which is associated with the degrading cell wall [57-61]. Usually, a typical secretory pathway in a cell is composed of at least two components, endoplasmic reticulum and Golgi apparatus, and two endomembrane systems, one for incoming, and another for outgoing traffic [62]. On the other hand, secretion of cellulase suggests the existence of three different mechanisms based on their subcellular location: a specific secretory pathway independent of cellulose, a secretory pathway, which is induced by cellulose, and a process that occurs irrespective of the carbon source [63].

Cellulase secretion needs to be induced, and this process involves the synthesis of new proteins for constructing secretory pathways. In A. niger, de Oliveira et al. [64] described the induction of 254 different predicted proteins related to the secretory pathway. Interestingly, the induction of proteins seemed to be carbon source-dependent. Similarly, studies in Clostridium thermocellum showed that endoglucanase activity was regulated by carbon source [65-68]. In A. nidulans, nonessential protein kinases and phosphatases were involved in the sensing of carbon and/or energetic status, and in the regulation of hydrolytic enzyme production [69]. As discussed above, cAMP controls a wide range of processes in the cell, including activity of kinase proteins. 
The $\Delta a c y 1$ strain exhibited an increase in the cellobiohydrolase expression. Interestingly, upregulation of cel7a and cel6a was more evident and the intracellular cAMP content was higher in the presence of sophorose than in the presence of other carbon sources tested. Furthermore, secretion of CEL7A was altered compared to the QM9414 parental strain. These results suggest that sophorose may be sensed by a different signaling pathway in the $\Delta a c y 1$ mutant strain, and that mutation of the acy 1 gene may alter the pattern of cellulases secretion in this strain. However, additional studies will be needed to identify potential kinase proteins involved in this process in $T$. reese $i$.

\section{Conclusions}

The present study contributes to a better understanding of the role of cAMP signaling pathway in the regulation of cellulase expression in $T$. reesei. Our results showed that cAMP effects are carbon source-dependent, with regulation of cellobiohydrolases more evidently affected in the presence of sophorose. Interestingly, cellulase secretion was altered in the $\Delta a c y 1$ mutant strain, in which cAMP synthesis is disrupted. Moreover, our study is the first report discussing a potential role for cAMP in the regulation of cellulase secretion in $T$. reesei. These results suggest that cAMP is an important signaling pathway involved in cellulase expression in $T$. reesei, but only in the presence of sophorose. These findings contribute to the understanding of the molecular mechanisms involved in the regulation of the processes of cellulolytic enzyme synthesis and secretion.

\section{Additional file}

Additional file 1: Figure S1. Growth profiles of T. reesei QM9414 and $\Delta a c y 1$ strains in cellulose (A) and glycerol (B) as carbon source. Error bars are represented from three biological replicates. No significant difference was showed in growth between $\triangle \mathrm{acy} 1$ mutant strain and the parental QM9414 (PDF $117 \mathrm{~kb}$ )

\section{Abbreviations}

ACY1: Adenylate cyclase; BSA: Bovine serum albumin; CAMP: Cyclic AMP; APR: Alcohol precipitation reagent; dbcAMP: Dibutyryl-CAMP; ECL: Enhanced chemiluminescence; MEX: malt extract; PBS: Phosphate buffered saline; PKA: Protein kinase A; qRT-PCR: Quantitative real time-PCR; SDS: Sodium dodecyl sulfate; TCA: Trichloroacetic acid; TTBS: Tris-buffered saline.

\section{Competing interests}

The authors declare that they have no competing interests.

\section{Authors' contributions}

KMVN performed the experimental design, laboratory experiments, and drafted the manuscript. MNC performed experimental design, and laboratory experiments. RGP drafted the manuscript. FCMN and RRA performed the experimental design, and laboratory experiments of the indirect immunofluorescence and confocal microscopy. RNS designed the project, supervised the research study, prepared/drafted the manuscript, and approved the final version to be published. All authors have read and approved the final manuscript.
Authors' information

Not applicable.

\section{Acknowledgments}

This work was supported by the Fundação de Amparo à Pesquisa do Estado de São Paulo (FAPESP) (Processes FAPESP 2013/03681-9; 2014/23653-2). We are grateful to Dr. Monika Schmoll (AIT Austrian Institute of Technology $\mathrm{GmbH}$ ) for providing the $\Delta a c y 1$ strain, Professor Maria Cristina Roque Antunes Barreira for providing laboratory infrastructure for the preparation of samples for indirect immunofluorescence and confocal microscopy. In addition, we are grateful to Elizabete Rosa and Vani Maria Alves for their technical assistance.

\section{Author details}

${ }^{1}$ Department of Biochemistry and Immunology, Ribeirão Preto Medical School, University of São Paulo, 14049-900 Ribeirão Preto, SP, Brazil. ${ }^{2}$ Department of Cell Biology and Molecular and Pathogenic Bioagents, Ribeirão Preto Medical School, University of São Paulo, 14049-900 Ribeirão Preto, SP, Brazil.

Received: 30 July 2015 Accepted: 25 September 2015 Published online: 30 September 2015

\section{References}

1. Schmoll M, Schuster A. Biology and biotechnology of Trichoderma. Appl Microbiol Biotechnol. 2010;87:787-99.

2. Saloheimo A, Henrissat B, Hoffrén AM, Teleman O, Penttilä M. A novel, small endoglucanase gene, egl5, from Trichoderma reesei isolated by expression in yeast. Mol Microbiol. 1994;13:219-28.

3. Martinez D, Berka RM, Henrissat B, Saloheimo M, Arvas M, Baker SE, et al.: Speaker: TzeFeng-Tian Advisor: Ruey-Shyang, Hseu, PhD Many evidences show the importance of bioethanol y Reduce dependent on imported petroleum y Lower environmental pollution y Cellulosic ethanol offers large reductions in green house gas (GHG) emi. 2008:553-560.

4. Dos Santos CL, Pedersoli WR, Antoniêto ACC, Steindorff AS, Silva-Rocha R, Martinez-Rossi NM, et al. Comparative metabolism of cellulose, sophorose and glucose in Trichoderma reesei using high-throughput genomic and proteomic analyses. Biotechnol Biofuels. 2014;7:41.

5. Antoniêto ACC, dos Santos Castro L, Silva-Rocha R, Persinoti GF, Silva RN Defining the genome-wide role of CRE1 during carbon catabolite repression in Trichoderma reesei using RNA-Seq analysis. Fungal Genet Biol. 2014;73:93-103.

6. Seiboth B, Ivanova C, Seidl-Seiboth V. Trichoderma reesei: A fungal enzyme producer for cellulosic biofuels. In: Marco Aurélio dos Santos Bernardes, editors. Biofuel Production—Recent Developments and Prospects. In Tech; 2011, p. 309-40.

7. Tisch D, Kubicek CP, Schmoll M. New insights into the mechanism of light modulated signaling by heterotrimeric G-proteins: ENVOY acts on gna1 and gna3 and adjusts CAMP levels in Trichoderma reesei (Hypocrea jecorina). Fungal Genet Biol. 2011;48:631-40.

8. Alspaugh JA, Pukkila-Worley R, Harashima T, Cavallo LM, Funnell D, Cox GM, et al. Adenylyl cyclase functions downstream of the Ga protein Gpa1 and controls mating and pathogenicity of Cryptococcus neoformans. Eukaryot Cell. 2002;1:75-84.

9. Casas-Flores S, Rios-Momberg M, Rosales-Saavedra T, Martínez-Hernández P, Olmedo-Monfil V, Herrera-Estrella A. Cross talk between a fungal blue-light perception system and the cyclic AMP signaling pathway. Eukaryot Cell. 2006;5:499-506.

10. Friedl MA, Kubicek CP, Druzhinina IS. Carbon source dependence and photostimulation of conidiation in Hpocrea atroviridis. Appl Environ Microbiol. 2008;74:245-50.

11. Lengeler KB, Davidson RC, D'souza C, Harashima T, Shen WC, Wang P, et al. Signal transduction cascades regulating fungal development and virulence. Microbiol Mol Biol Rev. 2000;64:746-85.

12. Mukherjee M, Mukherjee PK, Kale SP. CAMP signalling is involved in growth, germination, mycoparasitism and secondary metabolism in Trichoderma virens. Microbiology. 2007;153:1734-42.

13. Sesták S, Farkas V. Metabolic regulation of endoglucanase synthesis in Trichoderma reesei: participation of cyclic AMP and glucose-6-phosphate. Can J Microbiol. 1993;39:342-7.

14. Dashtban M, Schraft H, Qin W. Fungal bioconversion of lignocellulosic residues; opportunities \& perspectives. Int J Biol Sci. 2009;5:578-95. 
15. Bermejo C, Haerizadeh F, Sadoine MSC, Chermak D, Frommer WB. Differential regulation of glucose transport activity in yeast by specific CAMP signatures. Biochem J. 2013;452:489-97.

16. Schmoll M, Kubicek CP. Regulation of Trichoderma cellulase formation: lessons in molecular biology from an industrial fungus. A review Acta Microbiol Immunol Hung. 2003;50:125-45.

17. Schuster A, Tisch D, Seidl-Seiboth V, Kubicek CP, Schmoll M. Roles of protein kinase $\mathrm{A}$ and adenylate cyclase in light-modulated cellulase regulation in Trichoderma reesei. Appl Environ Microbiol. 2012;78:2168-78.

18. Schmoll M, Schuster A, Silva RDN, Kubicek CP. The G-alpha protein GNA3 of Hypocrea jecorina (Anamorph Trichoderma reesei) regulates cellulase gene expression in the presence of light. Eukaryot Cell. 2009;8:410-20.

19. Steiger MG, Mach RL, Mach-Aigner AR. An accurate normalization strategy for RT-qPCR in Hypocrea jecorina (Trichoderma reesei). J Biotechnol. 2010;145:30-7.

20. Simões AES, Pereira DM, Amaral JD, Nunes AF, Gomes SE, Rodrigues PM, et al. Efficient recovery of proteins from multiple source samples after TRIzol $\left({ }^{\circledast}\right)$ or TRIzol( $\left.{ }^{\circledast}\right)$ LS RNA extraction and long-term storage. BMC Genomics. 2013;14:181.

21. Livak KJ, Livak KJ, Schmittgen TD, Schmittgen TD. Analysis of relative gene expression data using real-time quantitative PCR and the 2(-Delta Delta C(T)) Method. Methods. 2001;25:402-8.

22. Castro LDS, Antoniêto ACC, Pedersoli WR, Silva-Rocha R, Persinoti GF, Silva RN. Expression pattern of cellulolytic and xylanolytic genes regulated by transcriptional factors XYR1 and CRE1 are affected by carbon source in Trichoderma reesei. Gene Expr Patterns. 2014;14:88-95.

23. Seibel C, Gremel G, Do Nascimento Silva R, Schuster A, Kubicek CP, Schmoll M. Light-dependent roles of the G-protein alpha subunit GNA1 of Hypocrea jecorina (anamorph Trichoderma reesei). BMC Biol. 2009;7:58.

24. Seibel C, Tisch D, Kubicek CP, Schmoll M. ENVOY is a major determinant in regulation of sexual development in Hypocrea jecorina (Trichoderma reesei) Eukaryot Cell. 2012;11:885-95.

25. Zhang J, Zhang Y, Zhong Y, Qu Y, Wang T. Ras GTPases modulate morphogenesis. Sporulation and cellulase gene expression in the cellulolytic fungus trichoderma reesei. PLoS One. 2012;7, e48786.

26. Wang $M$, Zhao Q, Yang J, Jiang B, Wang F, Liu K, et al. A mitogen-activated protein kinase $T m k 3$ participates in high osmolarity resistance, cell wall integrity maintenance and cellulase production regulation in Trichoderma reesei. PLoS One. 2013;8.

27. Wang M, Dong Y, Zhao Q, Wang F, Liu K, Jiang B, et al. Identification of the role of a MAP kinase Tmk2 in Hypocrea jecorina (Trichoderma reesei). Sci Rep. 2014:4:6732.

28. D'Souza CA, Heitman J. Conserved CAMP signaling cascades regulate fungal development and virulence. FEMS Microbiol Rev. 2001;25:349-64.

29. Rolland F, Winderickx J, Thevelein JM. Glucose-sensing mechanisms in eukaryotic cells. Trends Biochem Sci. 2001;310-317.

30. Palecek SP, Parikh AS, Kron SJ. Sensing, signalling and integrating physical processes during Saccharomyces cerevisiae invasive and filamentous growth. Microbiology. 2002;148:893-907.

31. Kronstad J, De Maria A, Funnell D, Laidlaw RD, Lee N, De Sá MM, et al. Signaling via CAMP in fungi: Interconnections with mitogen-activated protein kinase pathways. Arch Microbiol. 1998;170:395-404.

32. Hatanaka M, Shimoda C. The cyclic AMP/PKA signal pathway is required for initiation of spore germination in Schizosaccharomyces pombe. Yeast. 2001;18:207-17.

33. Bruno KS, Aramayo R, Minke PF, Metzenberg RL, Plamann M. Loss of growth polarity and mislocalization of septa in a Neurospora mutant altered in the regulatory subunit of CAMP-dependent protein kinase. EMBO J. 1996;15:5772-82

34. Shimizu K, Keller NP. Genetic involvement of a CAMP-dependent protein kinase in a $\mathrm{G}$ protein signaling pathway regulating morphological and chemical transitions in Aspergillus nidulans. Genetics. 2001;157:591-600.

35. Fillinger S, Chaveroche MK, Shimizu K, Keller N, D'Enfert C. CAMP and ras signalling independently control spore germination in the filamentous fungus Aspergillus nidulans. Mol Microbiol. 2002:44:1001-16

36. Saudohar M, Bencina M, van de Vondervoort PJl, Panneman H, Legisa M, Visser J, et al. Cyclic AMP-dependent protein kinase is involved in morphogenesis of Aspergillus niger. Microbiology. 2002;148(Pt 8):2635-45.

37. Rocha CR, Schröppel K, Harcus D, Marcil A, Dignard D, Taylor BN, et al. Signaling through adenylyl cyclase is essential for hyphal growth and virulence in the pathogenic fungus Candida albicans. Mol Biol Cell. 2001;12:3631-43.

38. Morosoli R, Durand S, Boucher F. Stimulation of xylanase synthesis in Cryptococcus albidus by cyclic AMP. FEMS Microbiol Lett. 1989;48:57-60.

39. el-Gogary S, Leite A, Crivellaro O, Eveleigh DE, el-Dorry H. Mechanism by which cellulose triggers cellobiohydrolase I gene expression in Trichoderma reesei. Proc Natl Acad Sci U S A. 1989;86:6138-41.

40. Mandels M, Reese ET. Induction of Cellulase in Fungi by Cellobiose. 1959;79:816-26.

41. Mandels M, Parrish FW, Reese ET. Sophorose as an inducer of cellulase in Trichoderma viride. J Bacteriol. 1962;83:400-8.

42. Sternberg D, Mandels GR. Induction of Cellulolytic Enzymes in Trichoderma reesei by Sophorose. J Bacteriol. 1979;139:761-9.

43. Hazlewood GP, Laurie Jl, Ferreira LM, Gilbert HJ. Pseudomonas fluorescens subsp. cellulosa: an alternative model for bacterial cellulase. J Appl Bacteriol. 1992;72:244-51.

44. Fang $X$, Yano $S$, Inoue $H$, Sawayama $S$. Lactose enhances cellulase production by the filamentous fungus Acremonium cellulolyticus. J Biosci Bioeng. 2008;106:115-20.

45. Sehnem NT, De Bittencourt LR, Camassola M, Dillon AJP. Cellulase production by Penicillium echinulatum on lactose. Appl Microbiol Biotechnol. 2006;72:163-7.

46. IImén M, Saloheimo A, Onnela ML, Penttilä ME. Regulation of cellulase gene expression in the filamentous fungus Trichoderma reesei. Appl Environ Microbiol. 1997:63:1298-306.

47. Fritscher C, Messner R, Kubicek CP. Cellobiose metabolism and cellobiohydrolase I biosynthesis by Trichoderma reesei. Exp Mycol. 1990;14:405-15.

48. Hu Y, Liu G, Li Z, Qin Y, Qu Y, Song X. G protein-CAMP signaling pathway mediated by PGA3 plays different roles in regulating the expressions of amylases and cellulases in Penicillium decumbens. Fungal Genet Biol. 2013:58-59:62-70.

49. Dong W, Yinbo Q, Peiji G. Regulation of cellulase synthesis in mycelial fungi: Participation of ATP and cyclic AMP. Biotechnol Lett. 1995; 17:593-8

50. Herrera-Herrera JA, Pérez-Avalos O, Salgado LM, Ponce-Noyola T. Cyclic AMP regulates the biosynthesis of cellobiohydrolase in Cellulomonas Xavigena growing in sugar cane bagasse. Arch Microbiol. 2009;191:745-50.

51. Rizzatti ACS, Freitas FZ, Bertolini MC, Peixoto-Nogueira SC, Terenzi HF, Jorge $J A$, et al. Regulation of xylanase in Aspergillus phoenicis: a physiological and molecular approach. J Ind Microbiol Biotechnol. 2008;35:237-44.

52. Schmoll M, Zeilinger S, Mach RL, Kubicek CP. Cloning of genes expressed early during cellulase induction in Hypocrea jecorina by a rapid subtraction hybridization approach. Fungal Genet Biol. 2004:41:877-87.

53. Carreras-Villasenor N, Sanchez-Arreguin JA, Herrera-Estrella AH. Trichoderma: sensing the environment for survival and dispersal. Microbiology. 2012;158:3-16

54. Farkaš V, Šesták S, Grešíak M, Kolarova N, Labudová I, Baucer Š. Induction of cellulase in Trichoderma reesei grown on lactose. Acta Biotechnol. 1987:7:425-9.

55. Montenecourt BS, Nhlapo SD, Trimino-Vazquez H, Cuskey S, Schamhart DH Eveleigh DE. Regulatory controls in relation to overproduction of fungal cellulases. Basic Life Sci. 1981;18:33-53.

56. Pakula TM, Uusitalo J, Saloheimo M, Salonen K, Aarts RJ, Penttilä M. Monitoring the kinetics of glycoprotein synthesis and secretion in the filamentous fungus Trichoderma reesei: Cellobiohydrolase I (CBHI) as a model protein. Microbiology. 2000;146:223-32.

57. Murashima K, Kosugi A, Doi RH. Synergistic effects on crystalline cellulose degradation between cellulosomal cellulases from Clostridium cellulovorans. J Bacteriol. 2002;184:5088-95

58. Koukiekolo R, Cho HY, Kosugi A, Inui M, Yukawa H, Doi RH. Degradation of corn fiber by Clostridium cellulovorans cellulases and hemicellulases and contribution of scaffolding protein CbpA. Appl Environ Microbiol. 2005;71:3504-11.

59. Tamaru Y, Miyake H, Kuroda K, Ueda M, Doi RH. Comparative genomics of the mesophilic cellulosome-producing Clostridium cellulovorans and its application to biofuel production via consolidated bioprocessing. Environ Technol. 2010;31:889-903.

60. Raman B, Pan C, Hurst GB, Rodriquez M, McKeown CK, Lankford PK, et al. Impact of pretreated Switchgrass and biomass carbohydrates on Clostridium 
thermocellum ATCC 27405 cellulosome composition: a quantitative proteomic analysis. PLoS ONE. 2009;4, e5271.

61. Ueda M, Goto T, Nakazawa M, Miyatake K, Sakaguchi M, Inouye K. A novel cold-adapted cellulase complex from Eisenia foetida: Characterization of a multienzyme complex with carboxymethylcellulase, beta-glucosidase, beta-1,3 glucanase, and beta-xylosidase. Comp Biochem Physiol - B Biochem Mol Biol. 2010;157:26-32.

62. Hilbi H, Haas A. Secretive bacterial pathogens and the secretory pathway. Traffic. 2012;13:1187-97.

63. Mcgavin M, Lam JOE, Forsberg CW. Regulation and Distribution of Fibrobacter succinogenes subsp. succinogenes 585 Endoglucanases. Appl Environ Microbiol. 2000;56:1235-44.

64. De Ferreira Oliveira JMP, Van Passel MWJ, Schaap PJ, De Graaff LH. Shotgun proteomics of aspergillus niger microsomes upon D-xylose inductions. Appl Environ Microbiol. 2010;76:4421-9.

65. Bayer EA, Kenig R, Lamed R. Adherence of Clostridium thermocellum to cellulose. J Bacteriol. 1983;156:818-27.

66. Lamed R, Setter E, Bayer EA. Characterization of a cellulose-binding, cellulase-containing complex in clostridium thermocellum. J Bacteriol. 1983;156:828-36.

67. Lamed R, Kenig R, Setter E, Bayer EA. Major characteristics of the cellulolytic system of Clostridium thermocellum coincide with those of the purified cellulosome. Enzyme Microb Technol. 1985;7:37-41.

68. Lamed R, Naimark J, Morgenstern E, Bayer EA. Specialized cell surface structures in cellulolytic bacteria. J Bacteriol. 1987;169:3792-800.

69. Brown NA de Gouvea PF, Krohn NG, Savoldi M, Goldman GH. Functional characterisation of the non-essential protein kinases and phosphatases regulating Aspergillus nidulans hydrolytic enzyme production. Biotechnol Biofuels. 2013;6:91.

\section{Submit your next manuscript to BioMed Central and take full advantage of:}

- Convenient online submission

- Thorough peer review

- No space constraints or color figure charges

- Immediate publication on acceptance

- Inclusion in PubMed, CAS, Scopus and Google Scholar

- Research which is freely available for redistribution 